Pacific Journal of Mathematic 


\title{
THE PALEY-WIENER THEOREM IN METRIC LINEAR SPACES
}

\author{
MaYnard G. Arsove
}

1. Introduction. By a basis in a topological linear space $\mathscr{T}$ we mean a sequence $\left\{x_{n}\right\}$ of points of $\mathscr{T}$ such that to every $x$ in $\mathscr{T}$ there corresponds a unique sequence $\left\{a_{n}\right\}$ of scalars for which

$$
x=\sum_{n=1}^{\infty} a_{n} x_{n} .
$$

Denoting the coefficient functionals here by $\varphi_{n}$, we can rewrite this as

$$
x=\sum_{n=1}^{\infty} \mathcal{P}_{n}(x) x_{n} .
$$

If it happens that all $\varphi_{n}$ are continuous on $\mathscr{T}$, the basis will be referred to as a Schauder basis. Every basis in a Fréchet space [14, pp. $59,110]$ is known to be a Schauder basis (see Newns [21], pp. 431-432), and it will be shown here that the same holds for bases in an arbitrary complete metric linear space over the real or complex field.

The classical Paley-Wiener theorem asserts that for $\mathscr{T}$ a Banach space, all sequences which sufficiently closely approximate bases must themselves be bases. A more precise statement of the theorem is obtained by replacing $\mathscr{C}$ in Theorem 1 by a Banach space $\mathscr{B}$.

The bibliography at the end of the present paper includes a chronological listing of articles on the Paley-Wiener theorem, and we give now a brief résumé of its history. As originally presented in 1934 by Paley and Wiener [1, p. 100], the theorem was derived specifically for the Hilbert space $L^{2}$. Then, in applying the theorem to the Pincherle basis problem [2, p. 469], Boas observed in 1940 that the proof of Paley and Wiener remains valid for Banach spaces. Boas also succeeded in simplifying a portion of the proof. However, the first really elementary proof of the theorem was published in 1949 by Schafke [8], to whom conclusion (3) is due. The remaining articles on the Paley-Wiener theorem deal mainly with various generalizations of condition (2.1) for Hilbert spaces.

From the viewpoint of modern functional analysis, the key to theorems of Paley-Wiener type lies in the inversion of an operator $I+T$ by means of a geometric series in $T$. This crucial observation was made by Buck [15, p. 410] in $1953 .^{1}$

Received July 28, 1958, and in revised form June 22, 1959. The research reported upon here was supported in part by the National Science Foundation.

1 The same technique was used also in [9], the author having been unaware of the earlier remarks of Buck. A further application (to generalized bases) appears in [12]. 
Our purpose in the present note is to utilize the operator technique in deriving a number of variants of the Paley-Wiener theorem. For reference, we begin by sketching a proof of the theorem itself for complete metric linear spaces. The ensuing variants then have in common the hypothesis that $\left\{x_{n}\right\}$ be a Schauder basis and $\left\{y_{n}\right\}$ a sequence triangular with respect to $\left\{x_{n}\right\}$. This is evidently motivated by the case of Pincherle bases in spaces of analytic functions (see, for example, [9]), and we conclude, in fact, with a generalization to arbitrary Fréchet spaces of the theorem of Boas [2, p. 447, Theorem 4.1] on Pincherle bases.

The author is indebted to Professor Robert C. James for reading the manuscript and suggesting a number of important simplifications. In particular, Theorem 2 replaces a weaker theorem of the original manuscript.

2. The proof for metric linear spaces. In what follows, we shall denote by $/ l$ a complete metric linear space over the real or complex field and employ the notation of Banach:

$$
\|x\|=\rho(x, 0)
$$

where $\rho$ is the metric on $\mathscr{l}$. It will be assumed further that $\rho$ is translation invariant. ${ }^{2}$

With these conventions the Paley-Wiener theorem can be formulated as

Theorem 1. Let $\left\{x_{n}\right\}$ and $\left\{y_{n}\right\}$ be sequences in , the and let $\lambda$ be a real number $(0<\lambda<1)$ such that

$$
\left\|\sum_{n=1}^{m} a_{n}\left(y_{n}-x_{n}\right)\right\| \leq \lambda\left\|\sum_{n=1}^{m} a_{n} x_{n}\right\|
$$

holds for all finite sequences $a_{1}, a_{2}, \cdots, a_{m}$ of scalars. Then

(1) if $\left\{x_{n}\right\}$ is total in $\mathbb{C l}$, so is $\left\{y_{n}\right\}$;

(2) if $\left\{x_{n}\right\}$ is a basis in $\mathscr{C l}$, so is $\left\{y_{n}\right\}$, and the coefficients in any expansion $\sum b_{n} y_{n}$ satisfy

$$
\left\|\sum_{n=1}^{\infty} b_{n} x_{n}\right\| \leq \frac{1}{1-\lambda}\left\|\sum_{n=1}^{\infty} b_{n} y_{n}\right\|
$$

(3) if $\left\{x_{n}\right\}$ is a basis in $\mathscr{l l}$, there exists an automorphism ${ }^{3} A$ on $\mathscr{C l}$ such that $y_{n}=A x_{n}(n=1,2, \cdots)$.

2 A translation-invariant metric yielding the original topology always exists (see, for example, [19, p. 34]).

3 The term automorphism is used to designate any linear homeomorphic mapping of the space onto itself. By the open mapping theorem [13, p. 41, Theorem 5] every oneto-one continuous linear mapping of $\mathscr{t h}$ onto itself is an automorphism on $\mathscr{t h}$. 
Proof. For convenience we consider first the case in which $\left\{x_{n}\right\}$ is a basis. Condition (2.1) then allows us to define a continuous linear operator $T$ on $\mathscr{l l}$ as

$$
T x=\sum_{n=1}^{\infty} \varphi_{n}(x) \cdot\left(y_{n}-x_{n}\right)
$$

and yields the inequality

$$
\left\|T^{n} x\right\| \leq \lambda^{n}\|x\| \quad(n=0,1, \cdots) .
$$

By comparison with the corresponding geometric series in $\lambda$ we infer convergence of the operator series

$$
U=\sum_{n=0}^{\infty}(-T)^{n}
$$

and obtain the inequality

$$
\|U x\| \leq(1-\lambda)^{-1}\|x\| .
$$

Hence, the linear operator $U$ is continuous on $-\mathbb{l l}$.

For any $x$ in $/ l$ the element $y$ of $/ l$ defined by $y=U x$ has the evident property that $x=(I+T) y$, where $I$ is the identity operator. From $y=\sum b_{n} x_{n}$ it therefore follows that $x=\sum b_{n} y_{n}$, and this proves that $\left\{y_{n}\right\}$ spans $/ \mathbb{C}$ in the infinite-series sense. That $\left\{y_{n}\right\}$ is linearly independent in the infinite-series sense can then be seen by rewriting (2.5) in the form (2.2). Assertions (2) and (3) are thereby established, the latter with $A$ taken as $I+T\left(=U^{-1}\right)$.

No essential change in the above argument is required to prove (1). We can clearly presume the $x_{n}$ to be finitely linearly independent and replace the infinite series in (2.3) by corresponding finite sums. Thus defined on a dense subset of $\mathscr{K}, T$ is then extended to all of $\mathscr{C l}$ in the usual fashion.

It should perhaps be mentioned that the automorphism $A$ in (3) is uniquely determined by the way it correlates the basis elements $x_{n}$ and $y_{n}$. In fact,

$$
A x=\sum_{n=1}^{\infty} \rho_{n}(x) y_{n}
$$

3. Coefficient functionals and coordinate subspaces. We recall that a Fréchet space is defined [14, pp. 59, 110] as a metrizable, complete, locally convex topological linear space over the real or complex field. Generalizing a theorem of Banach, Newns has shown [21, pp. 431-432] that for bases in Fréchet spaces the coefficient functionals $\varphi_{n}$ are always continuous. This can, however, be carried one step farther by discarding the hypothesis of local convexity. 
Such is the content of

THEOREM 2. Every basis in $\mathscr{C}$ is a Schauder basis.

Proof. As observed in footnote 2, there is no loss of generality in taking the metric $\rho$ on $\mathscr{C l}$ to be translation invariant. Having done this, we can conveniently make use of the functional $\|x\|=\rho(x, 0)$.

Let $\left\{x_{n}\right\}$ be a basis in $\mathscr{C}$, so that for each $x$ in $\mathscr{C}$ we have the expansion (1.1), or equivalently

$$
\lim _{m \rightarrow \infty}\left\|x-\sum_{n=1}^{m} \varphi_{n}(x) x_{n}\right\|=0 \text {. }
$$

Since this yields boundedness in $m$ (for fixed $x$ ) of

$$
\left\|\sum_{n=1}^{m} \varphi_{n}(x) x_{n}\right\|
$$

the quantity

$$
\|x\|^{\prime}=\sup _{m \geq 1}\left\|\sum_{n=1}^{m} \varphi_{n}(x) x_{n}\right\|
$$

is always finite. Thus, $\rho^{\prime}(x, y)=\|x-y\|^{\prime}$ defines a translation-invariant metric $\rho^{\prime}$ on $\mathscr{C l}$ with the property that $\rho(x, y) \leq \rho^{\prime}(x, y$ for all $x, y$ in $\mathscr{M}$.

It is immediate from (3.1) that

$$
\left\|\varphi_{n}(x) x_{n}\right\| \leq 2\|x\|^{\prime} \quad(n=1,2, \cdots),
$$

and the corollary to Proposition 2, pp. 25-26, of Bourbaki [14] then ensures that each $\rho_{n}$ is continuous in the metric $\rho^{\prime}$. The proof will be completed by showing that $\rho$ and $\rho^{\prime}$ define the same topology on $\mathscr{M}$.

We establish, first of all, that $\mathscr{C}$ is complete in the metric $\rho^{\prime}$. To this end, let $\left\{z_{k}\right\}$ be a Cauchy sequence in the metric $\rho^{\prime}$. From (3.2) and the result of Bourbaki just cited it follows that, for each $n$, $\left\{\varphi_{n}\left(z_{k}\right)\right\}_{k=1}^{\infty}$ is a Cauchy sequence of scalars and therefore converges to some scalar $c_{n}$. Now, given $\varepsilon>0$, there exists a positive integer $N$ such that $\left\|z_{j}-z_{k}\right\|^{\prime} \leq \varepsilon$ for $j, k>N$. For arbitrary positive integers $m$ and $m^{\prime} \leq m$ we thus have

$$
\left\|\sum_{n=m}^{m}\left[\Phi_{n}\left(z_{j}\right)-\varphi_{n}\left(z_{k}\right)\right] x_{n}\right\| \leq 2 \varepsilon \quad(j, k>N),
$$

which yields in the limit as $j \rightarrow \infty$

$$
\left\|\sum_{n=m^{\prime}}^{m} c_{n} x_{n}\right\| \leq 2 \varepsilon+\left\|\sum_{n=m^{\prime}}^{m} \phi_{n}\left(z_{k}\right) x_{n}\right\|
$$

The $\rho$-convergence of $\sum \varphi_{n}\left(z_{k}\right) x_{n}$ (for fixed $k$ ) gives rise to a Cauchy 
condition on its partial sums and thereby on the partial sums of $\sum c_{n} x_{n}$. Hence, $\sum c_{n} x_{n}$ converges $(\rho)$ to some point $z$ of $\mathscr{l l}$. Taking $m^{\prime}=1$ in (3.3) and passing to the limit on $j$, we arrive at

$$
\left\|z-z_{k}\right\|^{\prime}=\sup _{m \geq 1}\left\|\sum_{n=1}^{m}\left[c_{n}-\varphi_{n}\left(z_{k}\right)\right] x_{n}\right\| \leq 2 \varepsilon(k>N) .
$$

That is, $\left\{z_{k}\right\}$ converges to $z$ in the metric $\rho^{\prime}$.

The remainder of the proof involves simply a routine application of a corollary of the open mapping theorem [13, p. 41, Theorem 6] to conclude that $\rho$ and $\rho^{\prime}$ define the same topology on . $/ l$.

Relative to a given basis $\left\{x_{n}\right\}$, a coordinate subspace of $\mathscr{L l}$ is defined as a subspace of the form $\left\{x: \varphi_{n}(x)=0\right.$ for $\left.n \in K\right\}$, where $K$ is some set of positive integers. The coordinate subspaces arising when $K$ consists of the first $k-1$ positive integers are of special interest in the sequel, and we denote them by $\mathscr{M}_{k}$. That is, for each positive integer $k$, $\mathscr{C}_{k}$ is the set of all elements of $\mathscr{L l}$ expressible as infinite linear combinations of the basis elements $x_{k}, x_{k+1}, \ldots$. For convenience, $\mathscr{C}_{k}$ will be referred to as a terminal coordinate subspace (or, more precisely, as the kth terminal coordinate subspace) of Ll. relative to $\left\{x_{n}\right\}$.

Since coordinate subspaces relative to Schauder bases are necessarily closed, we have

CoRollary 2.1. All coordinate subspaces of Mll are closed.

4. Some variants of the Paley-Wiener theorem. A sequence $\left\{y_{n}\right\}$ in $\mathscr{C l}$ will be called triangular with respect to a basis $\left\{x_{n}\right\}$ provided that each $y_{n}$ has the representation

$$
y_{n}=x_{n}+\sum_{i=n+1}^{\infty} \varphi_{i}\left(y_{n}\right) x_{i} .
$$

In the present section we shall be concerned with the problem of determining conditions under which $\left\{y_{n}\right\}$ will itself be a basis in $\mathscr{C}$. This arises as a natural analogue of the Pincherle basis problem, and our methods here have much in common with those of [9].

We take advantage of the following special properties of triangular sequences.

Lemma 1. Let $\left\{x_{n}\right\}$ be a basis in $\mathscr{N}$, and let $\mathscr{N}_{k}$ be a terminal coordinate subspace of $\mathscr{C l}$ relative to $\left\{x_{n}\right\}$. If $\left\{y_{n}\right\}$ is a sequence in $\mathscr{C l}$ triangular with respect to $\left\{x_{n}\right\}$, then

(1) $\left\{y_{n}\right\}$ is linearly independent in the infinite-series sense, and

(2) for $\left\{y_{n}\right\}_{n=k}^{\infty}$ to be a basis in $\mathscr{N}_{k}$ it is necessary and sufficient that $\left\{y_{n}\right\}_{n=1}^{\infty}$ be a basis in $\mathscr{C l}$. 
Proof. To show that $\left\{y_{n}\right\}$ is linearly independent in the infiniteseries sense, we suppose that

$$
\sum_{n=1}^{\infty} b_{n} y_{n}=0
$$

Then, from (4.1) and the fact that $\mathscr{C}_{2}$ is closed, it is immediate that $b_{1} x_{1}+z_{2}=0$, where $z_{2}$ is some point in $\mathscr{L}_{2}$. Hence $b_{1}=0$, and an obvious inductive argument establishes $b_{n}=0(n=1,2, \cdots)$.

The second assertion is dealt with similarly. Let $\left\{y_{n}\right\}_{n=k}^{\infty}$ be a basis in $\mathscr{T}_{k}$, and let $y$ be any element of $\mathscr{C}$. It is evident that, for a suitably chosen scalar $b_{1}$, the point $y-b_{1} y_{1}$ will lie in $\mathscr{L}_{2}$. Proceeding inductively, we then see that there exist scalars $b_{1}, b_{2}, \cdots, b_{k-1}$ yielding

$$
y-\sum_{n=1}^{k-1} b_{n} y_{n} \in \mathscr{M}_{k} \text {. }
$$

Consequently, $\left\{y_{n}\right\}_{n=1}^{\infty}$ spans $\mathscr{C l}$ in the infinite-series sense and is therefore a basis in $\mathscr{l}$. The converse in (2) is trivial.

This leads to our first variant of Theorem 1.

THeOREM 3. Let $\left\{x_{n}\right\}$ be a basis in $\mathscr{M l}$ and $\left\{y_{n}\right\}$ a sequence triangular with respect to $\left\{x_{n}\right\}$. If there exist a positive number $\lambda<1$ and a positive integer $k$ such that

$$
\left\|\sum_{n=k}^{m} a_{n}\left(y_{n}-x_{n}\right)\right\| \leq \lambda\left\|\sum_{n=k}^{m} a_{n} x_{n}\right\|
$$

holds for all finite sequences $a_{k}, a_{k+1}, \cdots, a_{m}$ of scalars, then

(1) $\left\{y_{n}\right\}$ is a basis in $\mathscr{M l}$, and

(2) there exists an automorphism $A$ on $\mathbb{l l}$ such that $y_{n}=A x_{n}$ $(n=1,2, \cdots)$.

Proof. For conclusion (1) we apply Theorem 1 to infer that $\left\{y_{n}\right\}_{n=k}^{\infty}$ is a basis in $\mathscr{L}_{k}$, and then invoke (2) of Lemma 1. Theorem 1 shows also that the mapping

$$
A_{k} x=\sum_{n=k}^{\infty} \phi_{n}(x) y_{n}
$$

is an automorphism on $\mathscr{C}_{k}$. We can obviously extend $A_{k}$ to a mapping $A$ of $\mathscr{C}$ into itself by setting

$$
A x=\sum_{n=1}^{\infty} \varphi_{n}(x) y_{n},
$$

and from the fact that $\left\{y_{n}\right\}$ is a basis in $\mathscr{C}$ it is then clear that $A$ maps $\mathscr{C}$ onto itself in one-to-one fashion. There remains simply to observe that the continuity of $A_{k}$ implies continuity of $A$, so that $A$ is 
an automorphism on $\mathscr{C l}$.

A further variant of the main theorem is at hand when $\left\{x_{n}\right\}$ is an absolutely o-convergent basis in $\mathscr{C l}$, that is, when $\left\{x_{n}\right\}$ is a basis for which all $x \in \mathscr{M l}$ satisfy

$$
\sum_{n=1}^{\infty}\left\|\varphi_{n}(x) x_{n}\right\|<+\infty \cdot{ }^{4}
$$

THEOREM 4. Let $\left\{x_{n}\right\}$ be an absolutely $\rho$-convergent basis in. Ill and $\left\{y_{n}\right\}$ a sequence triangular with respect to $\left\{x_{n}\right\}$. If

$$
\limsup _{n \rightarrow \infty}\left\{\sup _{a \neq 0} \frac{\sum_{i=n+1}^{\infty}\left\|a \varphi_{i}\left(y_{n}\right) x_{i}\right\|}{\left\|a x_{n}\right\|}\right\}<1 \quad(a, \text { scalar })
$$

then

(1) $\left\{y_{n}\right\}$ is an absolutely $\rho$-convergent basis in $\mathscr{L l}$, and

(2) there exists an automorphism $A$ on . Ll such that $y_{n}=A x_{n}$ $(n=1,2, \cdots)$.

Proof. We first remetrize $\mathscr{C l}$ by setting $\rho^{\prime}(x, y)=\|x-y\|^{\prime}$, where

$$
\|x\|^{\prime}=\sum_{n=1}^{\infty}\left\|\varphi_{n}(x) x_{n}\right\|
$$

for all $x$ in $\mathscr{C}$. Obviously $\rho^{\prime}$ is translation invariant, and $\|x\| \leq\|x\|^{\prime}$.

Condition (4.3) can now be restated as follows: there exist a positive number $\lambda<1$ and a positive integer $k$ such that

$$
\left\|a\left(y_{n}-x_{n}\right)\right\|^{\prime} \leq \lambda\left\|a x_{n}\right\|^{\prime}
$$

holds for $n \geq k$ and all scalars $a$. This, together with (4.4), yields the inequality (4.2) in the metric $\rho^{\prime}$. Hence, $\left\{y_{n}\right\}$ is a basis in $\mathscr{C}$, and there exists an automorphism $A$ on $\mathscr{l}$ such that $y_{n}=A x_{n}(n=1,2$, $\cdots)$. It follows that, for arbitrary scalar sequences $\left\{b_{n}\right\}$, convergence of the series $\sum b_{n} y_{n}$ implies convergence (and thereby absolute $\rho$-convergence) of the series $\sum b_{n} x_{n}$. Since (4.5) results in

$$
\left\|b_{n} y_{n}\right\| \leq(1+\lambda)\left\|b_{n} x_{n}\right\|
$$

for $n \geq k$, we see that $\left\{y_{n}\right\}$ is, in fact, an absolutely $\rho$-convergent basis in $\mathscr{C l}$. This completes the proof.

As noted in the derivation, there is no real loss of generality in requiring that

$$
\left\|\sum_{n=1}^{\infty} \varphi_{n}(x) x_{n}\right\|=\sum_{n=1}^{\infty}\left\|\varphi_{n}(x) x_{n}\right\|
$$

${ }^{4}$ In metric linear spaces the notion of absolute $\rho$-convergence coincides with that of absolute convergence as defined by Day [16, pp. 11, 59] in terms of the Minkowski functional. Here, absolutely convergent bases are defined only for Fréchet spaces (see \&5). 
for all $x$ in $\mathscr{C}$. Whenever the metric $\rho$ and the basis $\left\{x_{n}\right\}$ are interrelated in this fashion, condition (4.3) assumes the simpler form

$$
\limsup _{n \rightarrow \infty}\left\{\sup _{a \neq 0} \frac{\left\|a\left(y_{n}-x_{n}\right)\right\|}{\left\|a x_{n}\right\|}\right\}<1 \quad \text { (a, scalar). }
$$

5. The case of Fréchet spaces. Proposition 6, p. 97, of [14] ensures that the topology on a Fréchet space $\mathscr{F}$ can be described by a sequence $\left\{\|\|_{q}\right\}$ of continuous semi-norms, and with no loss of generality this sequence will be taken as monotone increasing (a condition automatically fulfilled in spaces of analytic functions). Thus, $\|x\|_{p} \leq\|x\|_{q}$ for $q>p$ and all $x \in \mathscr{F}$; and convergence in $\mathscr{F}$ is equivalent to convergence with respect to each of the semi-norms \|\|$_{q}$. The topology on $\mathscr{F}$ is then that of the translation-invariant metric

$$
\rho(x, y)=\sum_{q=1}^{\infty} \frac{1}{2^{q}} \frac{\|x-y\|_{q}}{1+\|x-y\|_{q}} .
$$

As we proceed to show, the Paley-Wiener theorem and its variants can be generalized in the case of Fréchet spaces by replacing the inequalities on the metric by corresponding inequalities on the semi-norms.

Theorem 5. Let $\left\{x_{n}\right\}$ and $\left\{y_{n}\right\}$ be sequences in a Fréchet space $\mathscr{F}$, and let $\left\{\lambda_{q}\right\}$ be a sequence of real numbers $\left(0<\lambda_{q}<1\right)$ such that

$$
\left\|\sum_{n=1}^{m} a_{n}\left(y_{n}-x_{n}\right)\right\|_{q} \leq \lambda_{q}\left\|\sum_{n=1}^{m} a_{n} x_{n}\right\|_{q} \quad(q=1,2, \cdots)
$$

holds for all finite sequences $a_{1}, a_{2}, \cdots, a_{m}$ of scalars. Then

(1) if $\left\{x_{n}\right\}$ is total in $\mathscr{F}$, so is $\left\{y_{n}\right\}$;

(2) if $\left\{x_{n}\right\}$ is a basis in $\mathscr{F}$, so is $\left\{y_{n}\right\}$, and the coefficients in any expansion $\sum b_{n} y_{n}$ satisfy

$$
\left\|\sum_{n=1}^{\infty} b_{n} x_{n}\right\|_{q} \leq \frac{1}{1-\lambda_{q}}\left\|\sum_{n=1}^{\infty} b_{n} y_{n}\right\|_{q} \quad(q=1,2, \cdots) ;
$$

(3) if $\left\{x_{n}\right\}$ is a basis in $\mathscr{F}$, there exists an automorphism $A$ on $\mathscr{F}$ such that $y_{n}=A x_{n}(n=1,2, \cdots)$.

Theorem 6. Let $\left\{x_{n}\right\}$ be a basis in a Fréchet space F and $\left\{y_{n}\right\}$ a sequence triangular with respect to $\left\{x_{n}\right\}$. If there exist positive integers $k_{q}$ and positive numbers $\lambda_{q}<1$ such that

$$
\left\|\sum_{n=k_{q}}^{m} a_{n}\left(y_{n}-x_{n}\right)\right\|_{q} \leq \lambda_{q}\left\|\sum_{n=k_{q}}^{m} a_{n} x_{n}\right\|_{q} \quad(q=1,2, \cdots)
$$

holds for all finite sequences $a_{k_{q}}, a_{k_{q}+1}, \cdots, a_{m}$ of scalars, then

(1) $\left\{y_{n}\right\}$ is a basis in $\mathscr{F}^{q}$, and 
(2) there exists an automorphism $A$ on $\mathscr{T}$ such that $y_{n}=A x_{n}$ $(n=1,2, \cdots)$.

The proof of Theorem 5 duplicates that of Theorem 1. The proof of Theorem 6 would likewise duplicate that of Theorem 3 if we knew that $\left\{k_{q}\right\}$ were bounded (so that in effect we could replace it by a single number $k$ ). Failing this, we use the following argument, based directly on the properties of the transformation $T$ of (2.3).

Convergence of the series

$$
T x=\sum_{n=1}^{\infty} \varphi_{n}(x)\left(y_{n}-x_{n}\right)
$$

is ensured by condition (5.2). In fact, if $x$ lies in the $k_{q}$ th terminal coordinate subspace $\mathscr{F}_{k_{q}}$, we have

$$
\|T x\|_{q} \leq \lambda_{q}\|x\|_{q} \quad(q=1,2, \cdots) .
$$

Since the complementary subspace corresponding to each $\mathscr{F}_{k_{q}}$ is finite dimensional, it follows that $T$ is continuous on $\mathscr{F} .5$

Now, taking account of the fact that $\mathscr{F}_{k}$ is closed, we verify at once that $x$ in $\mathscr{F}_{k-1}$ implies $T x$ in $\mathscr{F}_{k}$. Hence, for arbitrary $x$ in $\mathscr{F}$, the point $T^{k-1} x$ must lie in $\mathscr{F}_{k}(k=1,2, \cdots)$. This result, combined with (5.3), leads to the inequality

$$
\left\|T^{n} x\right\|_{q} \leq\left(\lambda_{q}\right)^{n-k_{q}}\left\|T^{k_{q}} x\right\|_{q} \quad(q=1,2, \cdots)
$$

for $n \geq k_{q}$ and all $x$ in $\mathscr{F}$. As in the proof of Theorem 1, it follows that the operator series

$$
U=\sum_{n=0}^{\infty}(-T)^{n}
$$

converges and that $U=(I+T)^{-1}$. From this we conclude that $A=$ $I+T$ is an automorphism on $\mathscr{F}$ carrying $x_{n}$ into $y_{n}(n=1,2, \cdots)$, and that $\left\{y_{n}\right\}$ is a basis in $\mathscr{F}$.

To frame an analogue of Theorem 4, we first define an absolutely convergent basis in the Fréchet space $\mathscr{F}$ as a basis $\left\{x_{n}\right\}$ such that

$$
\sum_{n=1}^{\infty}\left\|\varphi_{n}(x) x_{n}\right\|_{q}<+\infty \quad(q=1,2, \cdots)
$$

for all $x$ in $\mathscr{F}{ }^{6}$

5 Any $x$ in $\mathscr{F}$ can be expressed as $x=x^{\prime}+x^{\prime \prime}$, where $x^{\prime}$ is the projection of $x$ on the complementary subspace to $\mathscr{F}_{k}$ and $x^{\prime \prime}$ is the projection of $x$ on $\mathscr{F}_{k}$. By continuity of the coefficient functionals, $x \rightarrow 0$ implies $x^{\prime} \rightarrow 0$ and thereby $x^{\prime \prime} \rightarrow 0$. Then $T x^{\prime} \rightarrow 0$ and $T x^{\prime \prime} \rightarrow 0$, so that $T x \rightarrow 0$.

${ }^{6}$ It is evident from [14, p. 101] that this definition is independent of the choice of semi-norm sequence from among those defining the topology on $\mathscr{F}$. 
THeOREm 7. Let $\mathscr{F}$ be a Fréchet space, $\left\{x_{n}\right\}$ an absolutely convergent basis in $\mathscr{F}$, and $\left\{y_{n}\right\}$ a sequence triangular with respect to $\left\{x_{n}\right\}$. If

$$
\limsup _{n \rightarrow \infty} \frac{\sum_{i=n+1}^{\infty}\left\|\varphi_{i}\left(y_{n}\right) x_{i}\right\|_{q}}{\left\|x_{n}\right\|_{q}}<1 \quad(q=1,2, \cdots),
$$

then

(1) $\left\{y_{n}\right\}$ is an absolutely convergent basis in $\mathscr{F}$, and

(2) there exists an automorphism $A$ on $\mathscr{F}$ such that $y_{n}=A x_{n}$ $(n=1,2, \cdots)$.

\section{Proof. Setting}

$$
\|x\|_{q}^{\prime}=\sum_{n=1}^{\infty}\left\|\varphi_{n}(x) x_{n}\right\|_{q} \quad(q=1,2, \cdots)
$$

for all $x$ in $\mathscr{T}$, we observe that $\left\{\|\|_{q}^{\prime}\right\}$ is an increasing sequence of semi-norms on $\mathscr{F}$. It thus defines a metric $\rho^{\prime}$ on $\mathscr{F}$ according to (5.1), and there is no difficulty in showing that $\rho^{\prime}$ yields the same topology as $\rho .^{7}$ Then, to each index $q$ there correspond a positive number $\lambda_{q}<1$ and a positive integer $k_{q}$ such that

$$
\left\|y_{n}-x_{n}\right\|_{q}^{\prime}<\lambda_{q}\left\|x_{n}\right\|_{q}^{\prime}
$$

holds for $n>k_{q}$. The additivity property (5.5) assures us also that (5.2) holds for the primed semi-norms, and the proof is completed just as in the case of Theorem 4 .

Again we note that the semi-norms on $\mathscr{F}$ can be required to have the additivity property

$$
\left\|\sum_{n=1}^{\infty} \varphi_{n}(x) x_{n}\right\|_{q}=\sum_{n=1}^{\infty}\left\|\varphi_{n}(x) x_{n}\right\|_{q} \quad(q=1,2, \cdots)
$$

relative to the absolutely convergent basis $\left\{x_{n}\right\}$. In terms of a "natural" sequence of semi-norms of this sort, condition (5.4) reduces to

$$
\limsup _{n \rightarrow \infty} \frac{\left\|y_{n}-x_{n}\right\|_{q}}{\left\|x_{n}\right\|_{q}}<1 \quad(q=1,2, \cdots) .
$$

It is readily seen that the coefficients for an element in a given basis are finite linear combinations of the coefficients in a basis triangular with respect to the given one. We have, in fact,

Lemma 2. Let $\left\{x_{n}\right\}$ be a basis in $\mathscr{C l}$ and $\left\{y_{n}\right\}$ a basis triangular with respect to $\left\{x_{n}\right\}$. If $x$ is an element of $/ l$ having expansions in

7 This argument appears also in the proof of Lemma 4 of [11]. 
the two bases as

$$
x=\sum_{n=1}^{\infty} a_{n} x_{n} \quad \text { and } \quad x=\sum_{n=1}^{\infty} b_{n} y_{n},
$$

then

$$
a_{1}=b_{1} \quad \text { and } \quad a_{n}=b_{n}+\sum_{k=1}^{n-1} b_{n-k_{i}} \mathcal{P}_{n}\left(y_{n-k}\right) \quad(n \geq 2)
$$

Proof. The expansion of $x$ in the basis $\left\{y_{n}\right\}$ appears as

$$
\begin{array}{r}
x=b_{1}\left[x_{1}+\mathcal{P}_{2}\left(y_{1}\right) x_{2}+\mathcal{P}_{3}\left(y_{1}\right) x_{3}+\cdots\right] \\
+b_{2}\left[x_{2}+\phi_{3}\left(y_{2}\right) x_{3}+\cdots\right] \\
+b_{3}\left[x_{3}+\cdots\right] \\
+\cdots .
\end{array}
$$

Since $\mathscr{L}_{2}$ is closed, it follows from the linear independence of $\left\{x_{n}\right\}$ that $a_{1}=b_{1}$. The fact that $\mathscr{L}_{3}$ is closed then results in $a_{2}=b_{2}+\mathscr{\rho}_{2}\left(y_{1}\right)$, and the general formula is obtained by induction. (Note that the proof in no way depends on rearrangement of the series.)

Using this lemma, we show how certain inequalities on the coefficients $a_{n}$ give rise to corresponding inequalities on the coefficients $b_{n}$. The underlying space will be taken as a Fréchet space $\mathscr{F}$, and $\left\{y_{n}\right\}$ will again be assumed to be a basis triangular with respect to the basis $\left\{x_{n}\right\}$.

Thus, let $x$ be an element of $\mathscr{F}$ having the expansion

$$
x=\sum_{n=1}^{\infty} a_{n} x_{n},
$$

and for each $q$ let $M_{q}$ be a constant such that

$$
\left|a_{n}\right| \leq \frac{M_{q}}{\left\|x_{n}\right\|_{q}} \quad(n=1,2, \cdots) .
$$

Constants of this sort always exist if the basis $\left\{x_{n}\right\}$ is absolutely convergent, since we can, for example, put $M_{q}=\sum\left\|a_{n} x_{n}\right\|_{q}$. (In spaces of analytic functions, where we have access to the Cauchy inequalities, the maximum modulus of course yields a better choice for $M_{q}$.) In similar fashion $H_{q}\left(y_{n}\right)$ will be taken as any constant for which

$$
\left|\varphi_{i}\left(y_{n}\right)\right| \leq H_{q}\left(y_{n}\right) \frac{\left\|x_{n}\right\|_{q}}{\left\|x_{i}\right\|_{q}} \quad(i \geq n+1) .
$$

Absolute convergence of $\left\{x_{n}\right\}$ again suffices to ensure the existence of such a constant, for example 


$$
H_{q}\left(y_{n}\right)=\frac{\sum_{i=n+1}^{\infty}\left\|\varphi_{i}\left(y_{n}\right) x_{i}\right\|_{q}}{\left\|x_{n}\right\|_{q}},
$$

and our remark on the case of analytic function spaces carries over.

Combined with the identities on the coefficients given in Lemma 2, the above inequalities provide the estimates

$$
\begin{aligned}
& \left|b_{1}\right| \cdot\left\|x_{1}\right\|_{q} \leq M_{q}, \\
& \left|b_{n}\right| \cdot\left\|x_{n}\right\|_{q} \leq M_{q}+\sum_{k=1}^{n-1} H_{q}\left(y_{n-k}\right)\left|b_{n-k}\right| \cdot\left\|x_{n-k}\right\|_{q} \quad(n \geq 2) .
\end{aligned}
$$

We apply now a procedure based on the techniques (due to Narumi [20]) used in proving Theorem 5 of [10]. With $\left\{B_{n}\right\}$ defined inductively according to the equations

$$
\begin{aligned}
& B_{1}\left\|x_{1}\right\|_{q}=M_{q}, \\
& B_{n}\left\|x_{n}\right\|_{q}=M_{q}+\sum_{k=1}^{n-1} B_{n-k} H_{q}\left(y_{n-k}\right)\left\|x_{n-k}\right\|_{q}
\end{aligned}
$$

it is readily verified that

$$
B_{n}\left\|x_{n}\right\|_{q}-B_{n-1}\left\|x_{n-1}\right\|_{q}=B_{n-1} H_{q}\left(y_{n-1}\right)\left\|x_{n-1}\right\|_{q} .
$$

Thus, for $n \geq 2$

$$
B_{n}\left\|x_{n}\right\|_{q}=\left[1+H_{q}\left(y_{n-1}\right)\right] B_{n-1}\left\|x_{n-1}\right\|_{q},
$$

so that

$$
\begin{aligned}
& B_{1}\left\|x_{1}\right\|_{q}=M_{q}, \\
& B_{n}\left\|x_{n}\right\|_{q}=M_{q} \prod_{k=1}^{n-1}\left[1+H_{q}\left(y_{k}\right)\right]
\end{aligned}
$$

There follows

Theorem 8. Let $\mathscr{F}$ be a Fréchet space, $x$ an element of $\mathscr{F},\left\{x_{n}\right\}$ a basis in $\mathscr{F}$, and $\left\{y_{n}\right\}$ a basis triangular with respect to $\left\{x_{n}\right\}$. Let us suppose further that there exist constants $M_{q}$ such that the coefficients in the expansion

$$
x=\sum_{n=1}^{\infty} a_{n} x_{n}
$$

satisfy

$$
\left|a_{n}\right| \leq \frac{M_{q}}{\left\|x_{n}\right\|_{q}} \quad(n=1,2, \cdots)
$$

for each index $q$, and that there exist constants $H_{q}\left(y_{n}\right)$ for which (5.6) holds. Then the coefficients in the expansion 


$$
x=\sum_{n=1}^{\infty} b_{n} y_{n}
$$

satisfy the inequalities

$$
\left|b_{1}\right| \leq \frac{M_{q}}{\left\|x_{1}\right\|_{q}}, \quad\left|b_{n}\right| \leq \frac{M_{q}}{\left\|x_{n}\right\|_{q}} \prod_{k=1}^{n-1}\left[1+H_{q}\left(y_{k}\right)\right] \quad(n \geq 2) .
$$

If in addition there exist constants $J_{q}$ such that

$$
\limsup _{n \rightarrow \infty} H_{q}\left(y_{n}\right)<J_{q} \quad(q=1,2, \cdots),
$$

then there also exist constants $K_{q}$ such that

$$
\left|b_{n}\right|<\left(1+J_{q}\right)^{n} \frac{K_{q}}{\left\|x_{n}\right\|_{q}} \quad(n=1,2, \cdots)
$$

for all $q$, and the constants $K_{q}$ are independent of $q$ whenever the same is true of $M_{q}, H_{q}\left(y_{n}\right)$, and $J_{q}$. In particular, condition (5.4) implies

$$
\left|b_{n}\right|<2^{n} \frac{K_{q}}{\left\|x_{n}\right\|_{q}}
$$

6. Concluding remarks. We begin by making explicit the specialization of Theorem 7 to spaces of analytic functions.

Thus, let $\Omega$ be a non-empty plane region, and fix $\left\{\Omega_{q}\right\}$ as any sequence of non-empty subregions of $\Omega$ such that $\bar{\Omega}_{q}$ is a compact subset of $\Omega_{q+1}(q=1,2, \cdots)$ and $\Omega=\cup \Omega_{q}$. The family of all functions $f$ analytic on $\Omega$, topologized by the sequence of semi-norms

$$
M_{q}(f)=\max _{\bar{\Omega} q}|f|,
$$

is a Fréchet space which we shall denote by $\mathscr{A}(\Omega)$.

Applied to $\mathscr{A}(\Omega)$, Theorem 7 yields the following variant of Theorem 2, p. 117, of Evgrafov [17]. ${ }^{8}$

THEOREM 9. Let $\left\{\alpha_{n}\right\}$ be an absolutely convergent basis in $\mathscr{\swarrow}(\Omega)$, and let $\left\{\beta_{n}\right\}$ be the triangular sequence defined by

$$
\beta_{n}(z)=\alpha_{n}(z)+\sum_{k=1}^{\infty} A_{n k} \alpha_{n+k}(z),
$$

where the $A_{n k}$ are any complex numbers for which the indicated series converge. If

8 Evgrafov's theorem, stated in terms of total systems, is given only for $\Omega$ simply connected and all $\alpha_{n}$ bounded on $\Omega$. Also, our condition of absolute convergence is replaced in the hypotheses of Evgrafov by the existence of a rather special sort of biorthogonal system. 


$$
\limsup _{n \rightarrow \infty} \sum_{k=1}^{\infty}\left|A_{n k}\right| \frac{M_{q}\left(\alpha_{n+k}\right)}{M_{q}\left(\alpha_{n}\right)}<1 \quad(q=1,2, \cdots),
$$

then $\left\{\beta_{n}\right\}$ is an absolutely convergent basis in $\mathscr{A}(\Omega)$, and there exists an automorphism on $\mathscr{A}(\Omega)$ carrying $\alpha_{n}$ into $\beta_{n}$ for each $n$.

A further specialization results in the theorem of Boas (cited in $\S 1$ ) on Pincherle bases in spaces of functions analytic on the discs $N_{R}(0)=$ $\{z:|z|<R\} \quad(0<R \leq+\infty)$. It is convenient here to let the index $n$ start with 0 and to put $\delta_{n}(z)=z^{n}(n=0,1, \cdots)$.

Corollary 9.1. (Boas). Let

$$
\alpha_{n}(z)=z^{n}\left(1+\sum_{k=1}^{\infty} A_{n k} z^{k}\right) \quad(n=0,1, \cdots),
$$

where the $A_{n k}$ are complex numbers, define a sequence in $\mathscr{A}\left(N_{R}(0)\right)$. If

$$
\limsup _{n \rightarrow \infty} \sum_{k=1}^{\infty}\left|A_{n k}\right| r^{k}<1
$$

for each $r<R$, then $\left\{\alpha_{n}\right\}$ is an absolutely convergent basis in $\mathscr{A}\left(N_{R}(0)\right)$, and there exists an automorphism $A$ on $\mathscr{A}\left(N_{R}(0)\right)$ such that $\alpha_{n}=A \delta_{n}$ $(n=0,1, \cdots){ }^{9}$

Returning to the case of general Fréchet spaces, we observe that the results of $\S 5$ remain valid if we assume only that the conditions on the semi-norms are satisfied for infinitely many indices $q$. In fact, the topology on $\mathscr{F}$ obviously is not affected if we replace the initial sequence of semi-norms by any subsequence of it.

Finally, we remark that when the underlying space is a Banach space, Theorems 4 and 7 coalesce. The common theorem is, however, somewhat restricted in scope, since every Banach space admitting an absolutely convergent basis is isomorphic to the space $l^{1}$ of absolutely summable sequences (see Karlin [18, p. 974]).

\section{BIBLIOGRAPHY}

\section{A. Chronological listing of articles on the Paley-Wiener theorem}

1. R. E. A. C. Paley and N. Wiener, Fourier transforms in the complex domain, New York, 1934.

2. R. P. Boas, Jr., Expansions of analytic functions, Trans. Amer. Math. Soc., 48 (1940), 467-487.

3. Y. Y. Tseng, On generalized biorthogonal expansions in metric and unitary spaces, Proc. Nat. Acad. Sci. U.S.A., 28 (1942), 35-43.

9 The theorem of Boas is enunciated in essentially this form by Evgrafov [17, p. 122] Theorem 2 of [9] states the result in a slightly restricted way by taking the limit as $r \rightarrow R$ of the left-hand member of (6.2). The proof in [9] is, however, valid in the present case. 
4. H. Pollard, Completeness theorems of Paley-Wiener type, Ann. of Math. (2), 45 (1944), 738-739.

5. B. Sz. Nagy, Expansion theorems of Paley-Wiener type, Duke Math. J., 14 (1947), 975-978.

6. S. H. Hilding, Note on completeness theorems of Paley-Wiener type, Ann. of Math. (2), 49 (1948), 953-955.

7. F. W. Schafke, Über einige unendliche lineare Gleichungssysteme, Math. Nachr., 3 (1949), 40-58.

8. — Das Kriterium von Paley und Wiener im Banachschen Raum, Math. Nachr., 3 (1949), 59-61.

\section{B. Other references}

9. M. G. Arsove, The Pincherle basis problem and a theorem of Boas, Math. Scand., 5 (1957), 271-275.

10. - Proper Pincherle bases in the space of entire functions, Quart. J. Math. (Oxford) (2), 9 (1958), 40-54.

11. - Similar bases and isomorphisms in Fréchet spaces, Math. Ann., 135 (1958), 283-293.

12. M. G. Arsove and R. E. Edwards, Generalized bases in topological linear spaces, Studia Math. (to appear).

13. S. Banach, Théorie des opérations linéaires, Varsovie, 1932.

14. N. Bourbaki, Espaces vectoriels topologiques (Ch. I, II), Paris, 1953.

15. R. C. Buck, Expansion theorems for analytic functions, Conference on functions of a complex variable, University of Michigan (1953), 409-419.

16. M. M. Day, Normed linear spaces, Berlin, 1958.

17. M. A. Evgrafov, The method of approximating systems in the space of analytic functions and its applications to interpolation, Trudy Moskovskovo Mat. Obshchestva, 5 (1956), 89-201. (Russian).

18. S. Karlin, Bases in a Banach space, Duke Math. J., 15 (1948), 971-985.

19. D. Montgomery and L. Zippin, Topological transformation groups, New York, 1955.

20. S. Narumi, A theorem on the expansion of analytic functions by infinite series, Tôhoku Math. J., 30 (1928), 441-444.

21. W. F. Newns, On the representation of analytic functions by infinite series. Phil. Trans. Royal Soc. London (A), 245 (1953), 429-468.

\section{Institut HenRI POINCARÉ}

AND

UNIVERSITY OF WASHINGTON 



\section{PACIFIC JOURNAL OF MATHEMATICS}

\section{EDITORS}

David Gilbarg

Stanford University

Stanford, California

F. H. Brownell

University of Washington

Seattle 5, Washington
A. L. Whiteman

University of Southern Californıa Los Angeles 7, California

L. J. PaIge

University of California

Los Angeles 24, California

\section{ASSOCIATE EDITORS}
E. F. BECKENBACH
T. M. CHERRY
D. DERRY

E. HEWITT
A. HORN
L. NACHBIN
M. OHTSUKA

H. L. ROYDEN

M. M. SCHIFFER
E. SPANIER

E. G. STRAUS

F. WOLF

\section{SUPPORTING INSTITUTIONS}

\author{
UNIVERSITY OF BRITISH COLUMBIA \\ CALIFORNIA INSTITUTE OF TECHNOLOGY \\ UNIVERSITY OF CALIFORNIA \\ MONTANA STATE UNIVERSITY \\ UNIVERSITY OF NEVADA \\ NEW MEXICO STATE UNIVERSITY \\ OREGON STATE COLLEGE \\ UNIVERSITY OF OREGON \\ OSAKA UNIVERSITY \\ UNIVERSITY OF SOUTHERN CALIFORNIA
}

\author{
STANFORD UNIVERSITY \\ UNIVERSITY OF TOKYO \\ UNIVERSITY OF UTAH \\ WASHINGTON STATE COLLEGE \\ UNIVERSITY OF WASHINGTON \\ AMERICAN MATHEMATICAL SOCIETY \\ CALIFORNIA RESEARCH CORPORATION \\ HUGHES AIRCRAFT COMPANY \\ SPACE TECHNOLOGY LABORATORIES \\ NAVAL ORDNANCE TEST STATION
}

Mathematical papers intended for publication in the Pacific Journal of Mathematics should be typewritten (double spaced), and the author should keep a complete copy. Manuscripts may be sent to any one of the four editors. All other communications to the editors should be addressed to the managing editor, L. J. Paige at the University of California, Los Angeles 24, California.

50 reprints per author of each article are furnished free of charge; additional copies may be obtained at cost in multiples of 50 .

The Pacific Journal of Mathematics is published quarterly, in March, June, September, and December. The price per volume (4 numbers) is $\$ 12.00$; single issues, $\$ 3.50$. Back numbers are available. Special price to individual faculty members of supporting institutions and to individual members of the American Mathematical Society: $\$ 4.00$ per volume; single issues, $\$ 1.25$.

Subscriptions, orders for back numbers, and changes of address should be sent to Pacific Journal of Mathematics, 2120 Oxford Street, Berkeley 4, California.

Printed at Kokusai Bunken Insatsusha (International Academic Printing Co., Ltd.), No. 6, 2-chome, Fujimi-cho, Chiyoda-ku, Tokyo, Japan.

PUBLISHED BY PACIFIC JOURNAL OF MATHEMATICS, A NON-PROFIT CORPORATION

The Supporting Institutions listed above contribute to the cost of publication of this Journal, but they are not owners or publishers and have no responsibility for its content or policies. 


\section{Pacific Journal of Mathematics}

\section{Vol. 10, No. $2 \quad$ October, 1960}

Maynard G. Arsove, The Paley-Wiener theorem in metric linear spaces ........

Robert (Yisrael) John Aumann, Acceptable points in games of perfect

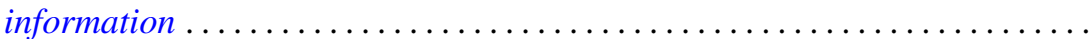

A. V. Balakrishnan, Fractional powers of closed operators and the semigroups generated by them ... . . . . . . . . . . . . . . . . . . . . . . . . . . . . 419

Dallas O. Banks, Bounds for the eigenvalues of some vibrating systems . . . . . 439

Billy Joe Boyer, On the summability of derived Fourier series . . . . . . . . . . . 475

Robert Breusch, An elementary proof of the prime number theorem with

remainder term ...................................

Edward David Callender, Jr., Hölder continuity of $n$-dimensional

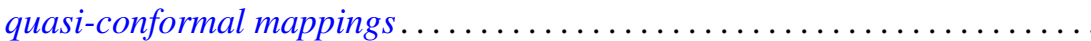

L. Carlitz, Note on Alder's polynomials ......................... 517

P. H. Doyle, III, Unions of cell pairs in $E^{3} \ldots \ldots \ldots \ldots \ldots \ldots \ldots \ldots \ldots \ldots \ldots \ldots . \ldots 21$

James Eells, Jr., A class of smooth bundles over a manifold . . . . . . . . . . . . 525

Shaul Foguel, Computations of the multiplicity function . . . . . . . . . . . . . . 539

James G. Glimm and Richard Vincent Kadison, Unitary operators in

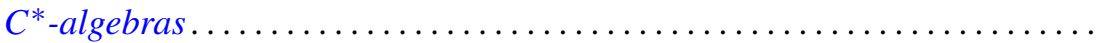

Hugh Gordon, Measure defined by abstract $L_{p}$ spaces . . . . . . . . . . . 557

Robert Clarke James, Separable conjugate spaces ....................

William Elliott Jenner, On non-associative algebras associated with bilinear forms

Harold H. Johnson, Terminating prolongation procedures

John W. Milnor and Edwin Spanier, Two remarks on fiber homotopy type .

Donald Alan Norton, A note on associativity . .

Ronald John Nunke, On the extensions of a torsion module.

Joseph J. Rotman, Mixed modules over valuations rings . . . . .

A. Sade, Théorie des systèmes demosiens de groupoï des . .

Wolfgang M. Schmidt, On normal numbers . .

661

Berthold Schweizer, Abe Sklar and Edward Oakley Thorp, The metrization of

statistical metric spaces

John P. Shanahan, On uniqueness questions for hyperbolic differential

equations

A. H. Stone, Sequences of coverings

Edward Oakley Thorp, Projections onto the subspace of compact operators

L. Bruce Treybig, Concerning certain locally peripherally separable spaces

Milo Wesley Weaver, On the commutativity of a correspondence and a

permutation

David Van Vranken Wend, On the zeros of solutions of some linear complex

differential equations. 